\title{
Resonance fluorescence of single molecules assisted by a plasmonic structure
}

\author{
Ying Gu, ${ }^{1}$ Lina Huang, ${ }^{2}$ Olivier J. F. Martin, ${ }^{2, *}$ and Qihuang Gong ${ }^{1, \dagger}$ \\ ${ }^{1}$ State Key Laboratory for Mesoscopic Physics, Department of Physics, Peking University, Beijing 100871, China \\ ${ }^{2}$ Nanophotonics and Metrology Laboratory, Swiss Federal Institute of Technology Lausanne (EPFL), EPFL-STI-NAM, ELG Station 11, \\ CH-1015 Lausanne, Switzerland
}

(Received 1 January 2010; revised manuscript received 5 April 2010; published 19 May 2010)

\begin{abstract}
The resonance fluorescence of a two-level single molecular system interacting with a plasmonic nanostructure is investigated. Specific regions of space are identified, where a balance exists between the near-field enhancement and the modification of the decay rate, such that the fluorescence spectrum of the molecule exhibits the Mollow triplet and the emission photons are antibunched. The utilization of such quantum phenomena at the vicinity of custom-designed plasmonic nanostructures paves the way for applications in nanoscale quantum devices and quantum information processing.
\end{abstract}

DOI: 10.1103/PhysRevB.81.193103

PACS number(s): 82.37.Vb, 73.20.Mf, 78.67.Bf

The interference of resonant transitions between atomic levels leads to many interesting quantum effects: Mollow absorption and triplet peaks, ${ }^{1}$ electromagnetically induced transparency, ${ }^{2}$ lasing without inversion, ${ }^{3}$ quantum switching, ${ }^{4}$ and spontaneous-emission cancellation. ${ }^{5}$ These effects are also exhibited by molecular systems. ${ }^{6-9}$ For example, photon antibunching was observed in the fluorescence spectrum of pentacene molecules trapped in a solid. ${ }^{7}$ Autler-Townes structures were reported in pump-probe experiments of single terrylene molecules at superfluid helium temperature. ${ }^{8}$ Mollow fluorescence triplet and coherent oscillations of emission photons were observed at low temperature $T=1.4 \mathrm{~K}$ by strongly focusing the far-field excitation onto the extinction area of molecules. ${ }^{9}$ In this work, instead of using far-field illumination, we utilize the dramatic nearfield enhancement provided at the vicinity of plasmonic structures to realize the strong coupling between single molecules and fields. Although molecules with complex vibration energy structures have a comparatively broad emission spectrum, the two-level approximation is perfectly well suited to describe them and has been widely used in the literature. ${ }^{7-9}$ We shall use this framework for this study.

When a quantum emitter is approaching a resonant plasmonic structure, it experiences a strong near-field enhancement as well as a significant modification of the decay rate of its transition channels. ${ }^{10}$ This modification of the population of excited state and decay rate can control the emission of fluorescent molecules and semiconductor quantum dots. ${ }^{11}$

Scanning near-field optical microscope probing of singlemolecule fluorescence indicates that near-field enhancement of resonant metallic nanoparticle leads to a change in intensity, lifetime, and spectrum of the fluorescence. ${ }^{12-14}$ Fluorescence quenching of dye molecules ${ }^{15}$ and changes in the shape of fluorescence spectra ${ }^{16}$ near gold nanoparticles have also been observed. When a single molecule is approaching a gold nanoparticle, a continuous transition from fluorescence enhancement to quenching is observed experimentally. ${ }^{12} \mathrm{Re}$ cently, the emission direction of a single molecule was experimentally realized by coupling the molecule to a specifically designed nanoantenna. ${ }^{17}$ Surface-dressed Bloch equations were furthermore used to study the fluorescence properties of two-level system near a metallic surface. ${ }^{18}$ However, the exact involvement of the large near field asso- ciated with surface plasmon resonances of isolated nanostructures in these quantum phenomena is not obvious.

The aim of this work is to introduce the quantum effects into a coupled system consisting of a quantum emitters and a custom-designed plasmon resonant structure. Using the Green's tensor technique, ${ }^{19}$ we have designed a silver nanostructure with four nanostrips to excite the fluorescence of single molecules. The optimal coupling coefficient, defined as the ratio of the near-field enhancement with the change in decay rate, can be reached in specific regions at the vicinity of the plasmonic structure, where a large near-field enhancement coexists with a decrease in the decay rate caused by cavity effects. ${ }^{20}$ Resonantly excited by enhanced near field, the fluorescence of single molecules exhibits the Mollow triplet and emission photons are antibunched. By adjusting the positions of single molecules, the splitting bands and spectral width of molecular fluorescence are dramatically modified. Utilizing near-field enhancement of surface plasmons to excite quantum emitters in a nanoscale provides a new tool to study quantum effects and shall open new perspectives to develop nanoscale quantum devices.

Let us first setup the coupled system consisting of a single molecule and a plasmonic nanostructure, within the general two-level framework. ${ }^{7-9}$ When light impinges on metallic nanostructures under suitable conditions collective oscillation of free electrons in the metal can be excited, leading to a strong near-field enhancement. The silver nanostructure composed of four nanostrips illustrated in Fig. 1(a) exhibits at resonance such a strong near-field enhancement near the gaps and extremities. Simultaneously, a cavity effect above the gaps decreases the decay rate of molecules located in these regions. If the Rabi frequency $\Omega_{R}$ is larger than the modified decay rate $\Gamma$, the resonant electromagnetic fields will dress the system well and the original two-level system will become a four-level one, ${ }^{21}$ as indicated in Fig. 1(c). As a consequence, Mollow triplet and photon antibunching of fluorescence can be observed in this system. ${ }^{1,9}$

As example, we choose dibenzanthanthrene (DBATT) molecules in a Shpol'skii matrix, which $0-0$ vibronic transition in $n$-tetradecane is at the wavelength of $\lambda=590 \mathrm{~nm}$ with a natural linewidth $\Gamma_{0}=17-20 \mathrm{MHz}$ and an excited-state lifetime of 9.7 ns. ${ }^{8,9}$ If the molecules are resonantly excited at $\lambda=590 \mathrm{~nm}$, they are well described by a two-level system. 

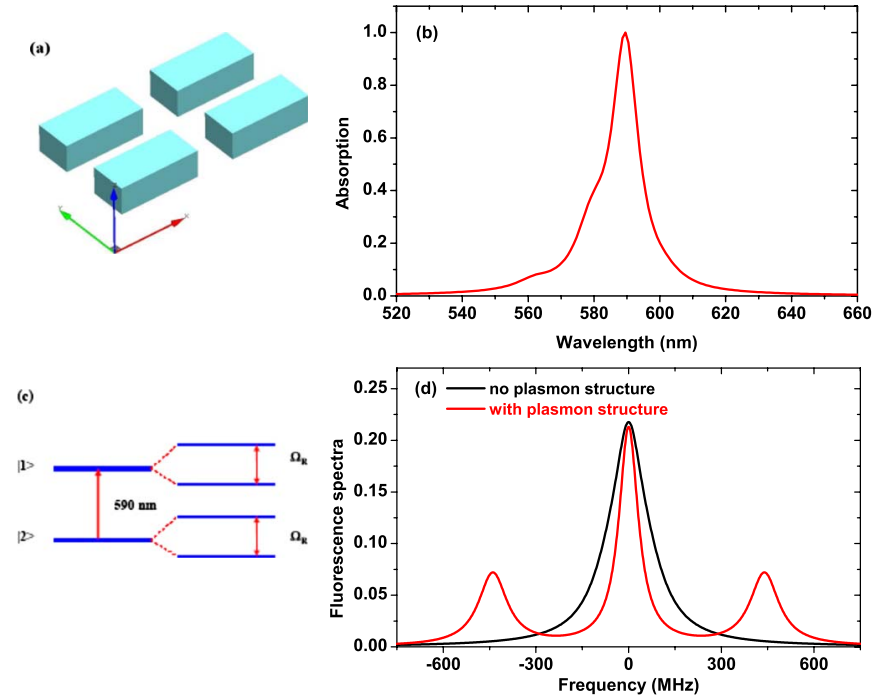

FIG. 1. (Color online) (a) Schematic and (b) absorption of a silver nanostructure composed of four nanostrips. Each nanostrip has dimensions $110 \times 50 \times 40 \mathrm{~nm}^{3}$ and each gap is $50 \mathrm{~nm}$ wide. The electric field is propagating along the $x$ direction and is polarized along the $z$ direction. The plasmon resonance wavelength is at $\lambda=590 \mathrm{~nm}$. (c) Dressed states diagram for a two-level DBATT molecule with Rabi splitting $\Omega_{R}$. The resonance wavelength between excited and ground levels is also $\lambda=590 \mathrm{~nm}$ and matches the plasmon resonance wavelength. (d) Fluorescence spectra for single molecules with and without plasmonic structure, computed in the middle of the $x y$ plane $50 \mathrm{~nm}$ above the metal surface.

Using the Green's tensor technique to compute the near field, ${ }^{19}$ we design a silver nanostructure composed of four nanostrips, such that its maximum field enhancement coincides with the absorption wavelength $\lambda=590 \mathrm{~nm}$, Fig. 1(b). A $10 \mathrm{~nm}$ mesh is used in the calculations. The nanostrips are $110 \times 50 \times 40 \mathrm{~nm}^{3}$ and the gaps are $50 \mathrm{~nm}$. The light is incident along the $z$ axis and polarized along the $x$ axis.

In absence of plasmonic nanostructures the molecule fluorescence when $\Omega_{R 0}=0.5 \times 2 \pi \Gamma_{0}$ exhibits only one single peak, as shown in Fig. 1(d). ${ }^{1}$ Under similar illumination conditions, the presence of the silver nanostructure increases the $x$ component of the electric field intensity in the middle of the $x y$ plane located $50 \mathrm{~nm}$ above the top nanostructure surface by a factor of 11.64 , i.e., $\Omega_{R}=11.644 \times \Omega_{R 0}$ and the decay rate becomes $\Gamma_{x x}=0.7068 \times \Gamma_{0}$. Furthermore, fluorescence linewidth is now reduced and two additional sidebands with about eight times $2 \pi \Gamma_{x x}$ offset appear in the fluorescence spectrum, Fig. 1(d). The following formulas are used for the calculations of the fluorescence: ${ }^{1}$

$$
\begin{aligned}
S(\vec{r}, \omega)= & \frac{I_{0}(\vec{r})}{8 \pi}\left[\frac{3 \Gamma / 4}{\left(\omega-\Omega_{R}-\omega_{0}\right)^{2}+(3 \Gamma / 4)^{2}}\right. \\
& \left.+\frac{\Gamma}{\left(\omega-\omega_{0}\right)^{2}+(\Gamma / 2)^{2}}+\frac{3 \Gamma / 4}{\left(\omega+\Omega_{R}-\omega_{0}\right)^{2}+(3 \Gamma / 4)^{2}}\right]
\end{aligned}
$$

and
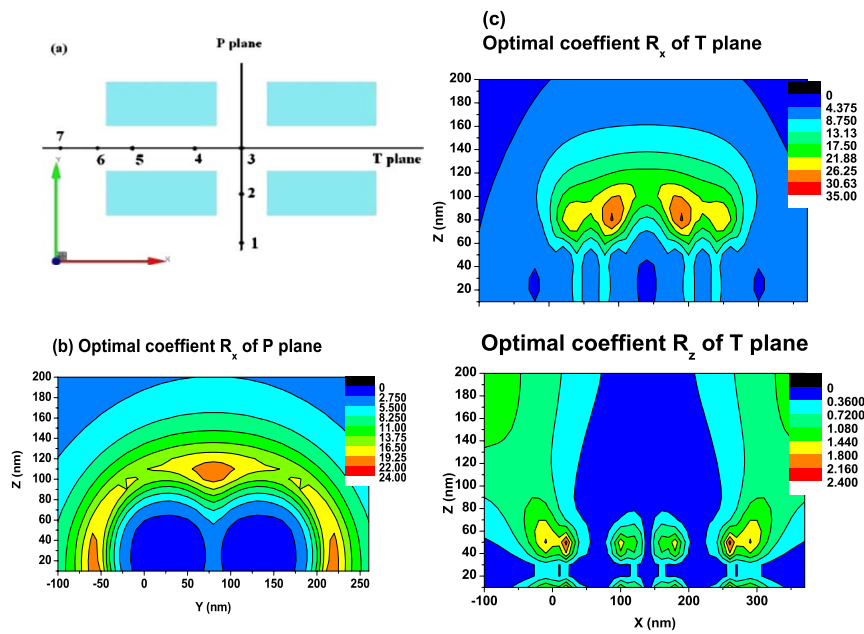

FIG. 2. (Color online) (a) Schematic of $P$ and $T$ planes. (b) Optimal coefficient $R_{x}$ for the $P$ plane and (c) $R_{x}$ and $R_{z}$ for the $T$ plane. The optimal values of $R_{x}$ are found within the volume located between 30 and $100 \mathrm{~nm}$ away from metal surface, where $R_{x}$ reaches values between 10 and 30 .

$$
g^{(2)}(\tau)=1-\left(\cos \mu \tau+\frac{3 \Gamma}{4 \mu} \sin \mu \tau\right) e^{-3 \Gamma \tau / 4}
$$

with

$$
\mu=\left(\Omega_{R}^{2}-\left(\frac{\Gamma}{4}\right)^{2}\right)^{1 / 2} .
$$

Near the plasmonic nanostructure, the decay rate $\Gamma_{0}$ for a dipole oriented in the $\alpha$ direction is modified as

$$
\Gamma_{\alpha \alpha}=3 \lambda \operatorname{Im}\left(G_{\alpha \alpha}\right) \Gamma_{0},
$$

where $G_{\alpha \alpha}$ is the Green's tensor. ${ }^{10}$

In order to find one or several regions around the nanostructure in which a molecule would produce strong resonance fluorescence, we must carefully consider the balance between the electric field enhancement and the decay-rate modification. By defining the optimal coefficient $R_{\alpha}=\frac{E_{\alpha} / E_{0}}{\Gamma_{\alpha \alpha} / \Gamma_{0}}$, we can find where it reaches its peak value. For this, we explore the optimal coefficients in both middle planes $[T$ plane and $P$ plane in Fig. 2(a), parallel to the $z$ axis and perpendicular to the $x y$ plane]. Molecules should not be too close to the metallic surfaces, at least more than $10 \mathrm{~nm}$ where the Forster energy transfer of electrons will begin. ${ }^{22}$ When the distance from the surface is smaller than $30 \mathrm{~nm}$, although the molecule is immersed in a strong near field, the optimal coefficient is still very small due to a huge decay rate, ${ }^{15}$ Fig. 2. In this case, the lifetime becomes very short, leading to fluorescence quenching ${ }^{15}$ and the emission spectrum is too wide to obtain a Mollow triplet. ${ }^{1}$ Conversely, when molecules are far away from the plasmonic structure (i.e., when the distance is larger than $100 \mathrm{~nm}$ ), although the modification of the decay rate is very small, the enhancement of the near field is also very small. Hence, it is difficult to produce a spectrum that exhibits quantum structures in these two extreme cases. However, the unique design of four nanostrips arranged together produces specific regions near the gaps, 

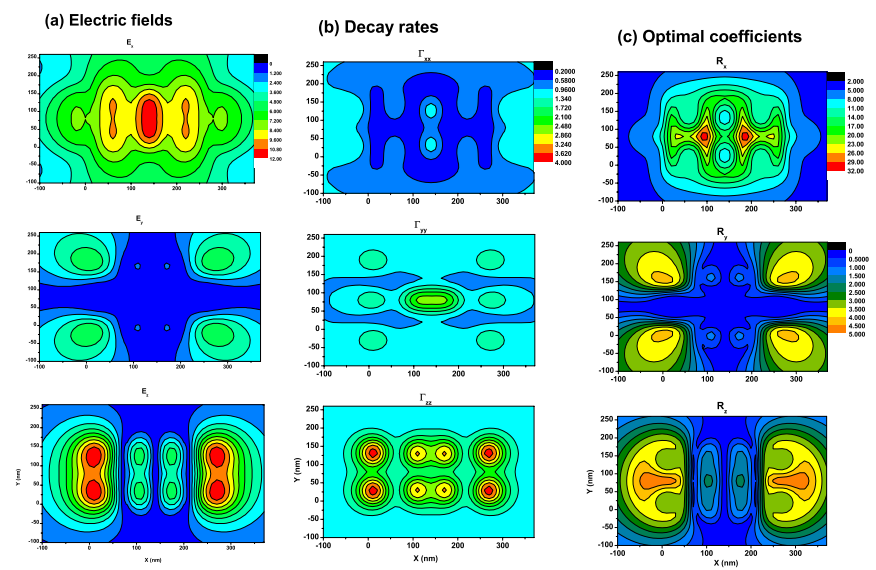

FIG. 3. (Color online) Near-field distribution, decay rate, and optimal coefficient for $x$ direction in the $x y P$ and $T$ planes located $50 \mathrm{~nm}$ from the metal surface. The near-field enhancement and the decrease in the decay rate produce an optimal region for $R_{x}$ above the gaps, where the resonance fluorescence of single molecules can be observed.

where the field is strongly enhanced and the decay rate marginally modified. We find that this occurs in a region 30-100 $\mathrm{nm}$ away from the metal surface, where $R(\alpha)$ reaches 30 , Fig. 2(c).

Let us now explore in detail the near-field enhancement and decay rate in the optimal region identified in Fig. 2, in the $x y$ plane $50 \mathrm{~nm}$ away from the metal surface, above the structure gap. The field enhancement in this plane reaches 12 times the illumination field in $x$ and $z$ directions, while the enhancement is only 5 in the $y$ direction, Fig. 3(a). In $x$ and $y$ directions, the enhancement of the decay rate is below two times that in a vacuum; while, in the $z$ direction it reaches four times, because a dipole normal to a metallic surface exhibits the largest decay rate, ${ }^{15}$ Fig. 3(b). In the $x$ direction, there even exist locations where $\Gamma_{x x}<1$, which correspond to a long lifetime of the molecule (i.e., a typical cavity effect ${ }^{20}$ ). A molecule located in this region will exhibit a much narrower resonance spectrum than in vacuum, Fig. 4(a). Furthermore, the optimal coefficient $R_{x}$ [shown in Fig. 3(c)] can reach a maximum of 30 with typical values between 10 and 30 in this plane. Hence, a fluorescing molecule located in this plane and polarized in $x$ direction will clearly exhibit resonant phenomena. Note, however, that the parameters $R_{y}$ and $R_{z}$ only reach the value of 5 . This is caused by the weak near-field enhancement in these directions. Hence molecules polarized in $y$ and $z$ directions will not exhibit resonance fluorescence.

As a matter of fact, the near field associated with the plasmon resonance occurring at the wavelength $\lambda=590 \mathrm{~nm}$ can be used to excite individual DBATT molecules located in the $x y$ plane, $50 \mathrm{~nm}$ away from the metal surface [their positions are shown in Fig. 2(a)]. Since the main contribution to fluorescence comes from the $x$-polarized dipoles, the contributions from molecules polarized in $y$ and $z$ directions are ignored. Figure 4 shows the resonance fluorescence spectra and their two photon correlation functions $g^{(2)}(\tau)$ for seven different molecule positions. The incident amplitude is normalized as $E_{0}=0.5$. Without a plasmon structure, a single-
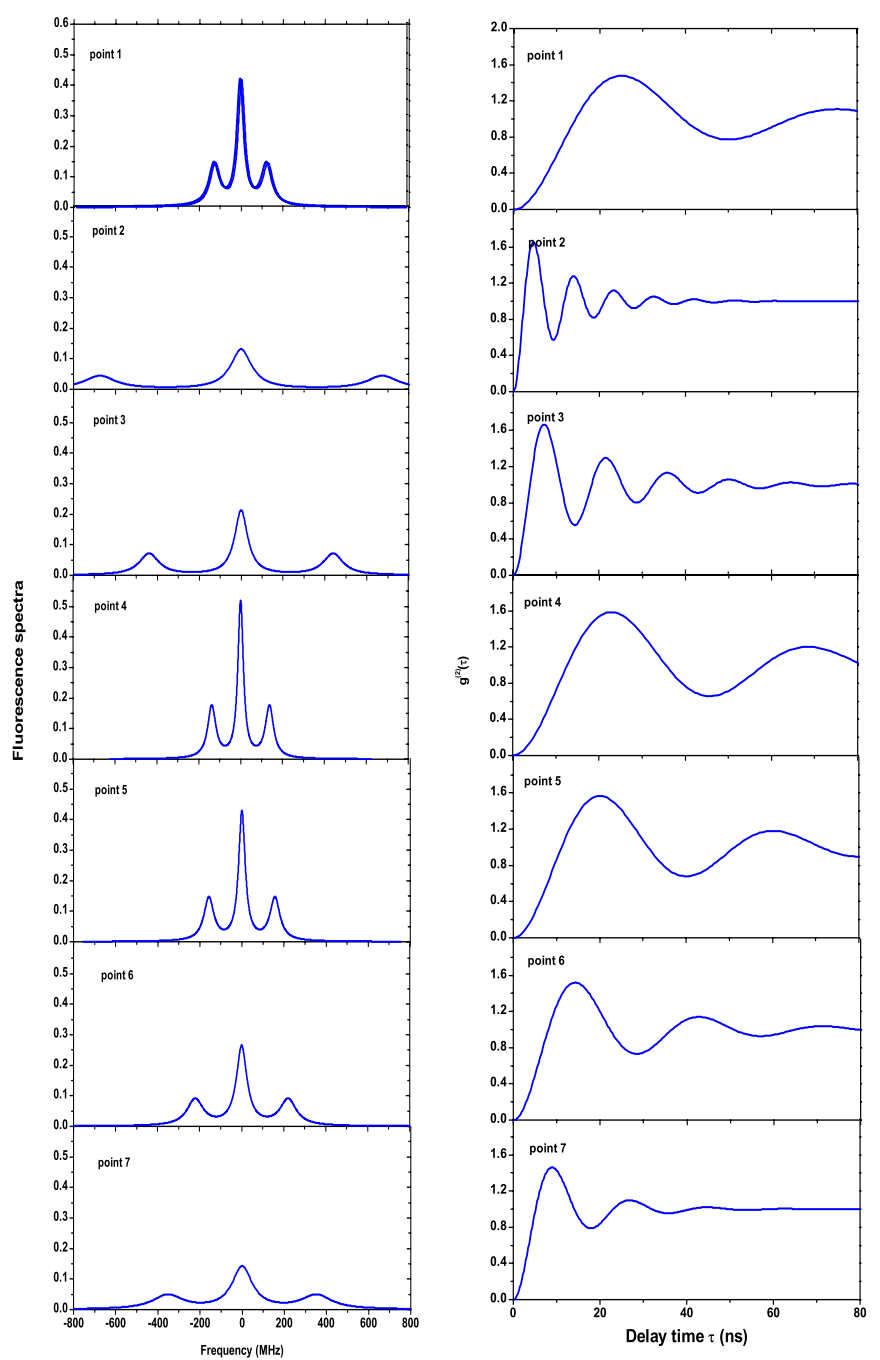

FIG. 4. (Color online) (a) Fluorescence power spectra and (b) second-order correlation $g^{2}(\tau)$ of emission photons for molecules located at the points 1, 2, 3, 4, 5, 6, and 7 in Fig. 2(a). The Mollow triplet and photon antibunching are clearly visible.

peak spectrum is obtained since $\Omega_{R 0}\left(=0.5 \times 2 \pi \Gamma_{0}\right)<2 \pi \Gamma_{0}$, Fig. 1(d). On the other hand, in the presence of the plasmon structure, $R_{x}$ is enhanced, which leads to $\Omega_{R} \gg 2 \pi \Gamma_{x x}$ and the Mollow triplet is obtained in these seven points. In Fig. 4(a), we observe that the linewidth for the emission spectra in points $1,4,5$, and 6 is narrower than that in vacuum due to the cavity effects ${ }^{20}$ while the linewidth for the points 2,3 , and 7 is approximately the same as that in vacuum. Hence, the sideband and linewidth of molecular resonance fluorescence are very sensitive to the location at the vicinity of the plasmonic structure. Figure 4(b) shows the corresponding second-order correlations of emission photons, where the antibunching phenomenon is clearly visible. As in Fig. 4(a), the molecules in points $1,4,5$, and 6 have a longer lifetime and those in points 2,3 , and 7 have a comparable lifetime than that in a vacuum. Therefore, thanks to appropriate design of the plasmonic structure and its cavity effect, it is possible to realize the Mollow triplet and photon antibunching in molecular fluorescence.

Let us briefly discuss some experimental aspects related 
to the possible demonstration of the effect studied in this Brief Report. Nowadays, metal nanostructures with dimensions controlled down to $10 \mathrm{~nm}$ can be realized using electron-beam lithography. ${ }^{23}$ The $0-0$ vibronic transition of DBATT fluorescent molecules can match the resonant wavelength of such plasmon nanostructures. These molecules can be diluted and spin coated over the nanostructures, and kept at a specific distance from the metal by first spin coating a dielectric spacer. Since the plasmon nanostructure enhances the electromagnetic field locally, the excitation of molecules is confined to small volumes, leading to a favorable signalto-noise ratio. Note that the molecular coating of the sample can help prevent silver oxidation. Finally, the substrate upon which the nanostructure will rest does redshift the resonance wavelength by and amount of $20-40 \mathrm{~nm}$. This can be compensated by shortening the nanostrips, or increasing their transverse sections, to maintain the resonance wavelength at $\lambda=590 \mathrm{~nm}^{24}$

In summary, we have found conditions where the Mollow triplet and photon antibunching of molecular fluorescence can be realized in plasmonic nanostructures. These effects require a subtle balance between the near-field enhancement and the modification of the lifetime caused by the nanostructure. In order to obtain high-quality resonance fluorescence spectra of single molecules, the designed system must satisfy two conditions: (i) the resonance wavelength of plasmonic structure must be similar to that of the molecules; (ii) the molecules must be located within optimal regions with a large near-field enhancement and a small modification of the decay rate. Let us mention that this is the first attempt to use the near-field enhancement of plasmonic nanostructures realize quantum effects. This work shall pave the way for applications in nanoscale quantum devices and quantum information processing.

This work was supported by the National Natural Science Foundation of China under Grants No. 10874004 and 10821062, National Key Basic Research Program under Grant No. 2007CB307001. Stimulating discussions with Xiaoyong Hu, Tiancai Zhang, Junxiang Zhang, B. S. Ham, and Christian Santschi are appreciated.

\footnotetext{
*olivier.martin@epfl.ch

†qhong@pku.edu.cn

${ }^{1}$ B. R. Mollow, Phys. Rev. 188, 1969 (1969); F. Y. Wu, S. Ezekiel, M. Ducloy, and B. R. Mollow, Phys. Rev. Lett. 38, 1077 (1977).

${ }^{2}$ S. E. Harris, Phys. Today 50 (7), 36 (1997).

${ }^{3}$ S. E. Harris, Phys. Rev. Lett. 62, 1033 (1989).

${ }^{4}$ B. S. Ham and P. R. Hemmer, Phys. Rev. Lett. 84, 4080 (2000).

${ }^{5}$ S. Y. Zhu and M. O. Scully, Phys. Rev. Lett. 76, 388 (1996); H. R. Xia, C. Y. Ye, and S. Y. Zhu, ibid. 77, 1032 (1996).

${ }^{6}$ J. Qi, G. Lazarov, X. Wang, L. Li, L. M. Narducci, A. M. Lyyra, and F. C. Spano, Phys. Rev. Lett. 83, 288 (1999).

${ }^{7}$ T. Basche, W. E. Moerner, M. Orrit, and H. Talon, Phys. Rev. Lett. 69, 1516 (1992).

${ }^{8}$ P. Tamarat, B. Lounis, J. Bernard, M. Orrit, S. Kummer, R. Kettner, S. Mais, and T. Basche, Phys. Rev. Lett. 75, 1514 (1995).

${ }^{9}$ G. Wrigge et al., Nat. Phys. 4, 60 (2008); I. Gerhardt, G. Wrigge, G. Zumofen, J. Hwang, A. Renn, and V. Sandoghdar, Phys. Rev. A 79, 011402(R)(2009).

${ }^{10}$ S. M. Barnett et al., J. Phys. B 29, 3763 (1996).

${ }^{11}$ J. R. Lakowicz, Plasmonics 1, 5 (2006).

${ }^{12}$ P. Anger, P. Bharadwaj, and L. Novotny, Phys. Rev. Lett. 96, 113002 (2006).

${ }^{13}$ S. Kuhn, U. Hakanson, L. Rogobete, and V. Sandoghdar, Phys.
}

Rev. Lett. 97, 017402 (2006).

${ }^{14}$ P. Bharadwaj and L. Novotny, Opt. Express 15, 14266 (2007).

${ }^{15}$ E. Dulkeith, A. C. Morteani, T. Niedereichholz, T. A. Klar, J. Feldmann, S. A. Levi, F. C. J. M. van Veggel, D. N. Reinhoudt, M. Moller, and D. I. Gittins, Phys. Rev. Lett. 89, 203002 (2002).

${ }^{16}$ M. Ringler, A. Schwemer, M. Wunderlich, A. Nichtl, K. Kurzinger, T. A. Klar, and J. Feldmann, Phys. Rev. Lett. 100, 203002 (2008).

${ }^{17}$ T. H. Taminiau et al., Nat. Photonics 2, 234 (2008).

${ }^{18}$ X. Huang, K. Lee, and T. F. George, J. Chem. Phys. 85, 567 (1986); X. Li and C. Gong, J. Phys. B 21, 1429 (1988); X. S. Li, D. L. Lin, and T. F. George, Phys. Rev. B 41, 8107 (1990).

${ }^{19}$ Y. Gu et al., EPL 83, 27004 (2008); O. J. F. Martin, C. Girard, and A. Dereux, Phys. Rev. Lett. 74, 526 (1995).

${ }^{20}$ J. Ye, D. W. Vernooy, and H. J. Kimble, Phys. Rev. Lett. 83, 4987 (1999).

${ }^{21}$ C. Cohen-Tannoudji, J. Dupont-Roc, and G. Grynberg, Atom Photon Interactions (Wiley, New York, 1992).

${ }^{22}$ B. Valeur, Molecular Fluorescence: Principles and Applications (Wiley-VCH, New York, 2001).

${ }^{23}$ W. Zhang et al., J. Phys. Chem. C 113, 14672 (2009).

${ }^{24}$ J. Li et al., J. Mod. Opt. 56, 1396 (2009). 